\title{
A Large-Eddy Simulation Study of Turbulent Flow Over Multiscale Topography
}

\author{
Feng Wan · Fernando Porté-Agel
}

Received: 1 March 2011 / Accepted: 22 July 2011 / Published online: 9 August 2011

(C) Springer Science+Business Media B.V. 2011

\begin{abstract}
Most natural landscapes are characterized by multiscale (often multifractal) topography with well-known scale-invariance properties. For example, the spectral density of landscape elevation fields is often found to have a power-law scaling behaviour (with a -2 slope on a log-log scale) over a wide span of spatial scales, typically ranging from tens of kilometres down to a few metres. Even though the effect of topography on the atmospheric boundary layer (ABL) has been the subject of numerous studies, few have focussed on multiscale topography. In this study, large-eddy simulation (LES) is used to investigate boundary-layer flow over multiscale topography, and guide the development of parametrizations needed to represent the effects of subgrid-scale (SGS) topography in numerical models of ABL flow. Particular emphasis is placed on the formulation of an effective roughness used to account for the increased aerodynamic roughness associated with SGS topography. The LES code uses the scale-dependent Lagrangian dynamic SGS model for the turbulent stresses and a terrain-following coordinate transformation to explicitly resolve the effects of the topography at scales larger than the LES resolution. The terrain used in the simulations is generated using a restricted solid-on-solid landscape evolution model, and it is characterized by a -2 slope of the elevation power spectrum. Results from simulations performed using elevation fields band-pass filtered at different spatial resolutions indicate a clear linear relation between the square of the effective roughness and the variance of elevation.
\end{abstract}

Keywords Large-eddy simulation $\cdot$ Multiscale complex terrain $\cdot$ Subgrid-scale topography

F. Wan

Saint Anthony Falls Laboratory, Department of Civil Engineering, University of Minnesota, Minneapolis, MN 55414, USA

F. Porté-Agel $(\varangle)$

School of Architecture, Civil and Environmental Engineering (ENAC), École Polytechnique Fédérale de Lausanne (EPFL), 1015 Lausanne, Switzerland

e-mail: fernando.porte-agel@epfl.ch 


\section{Introduction}

The interaction between natural forces (e.g., geological uplift, overland water flow and erosion) and the earth's surface results in various geomorphic processes that shape landscapes at a wide range of spatial and temporal scales. As a result, natural landscapes exhibit multiscale (often multifractal) structure, a high degree of self-similarity, and well-known scaling properties (e.g., Rodriguez-Iturbe and Rinaldo 1997). For example, the power spectra of linear transects in many natural landscapes, when plotted on a log-log scale, show a clear -2 slope over a wide range of spatial scales, from a few metres up to tens of kilometres (e.g., Huang and Turcotte 1989; Passalacqua et al. 2006). Undoubtedly, the scaling properties of surface elevation have effects on the multiscale properties of turbulent atmospheric boundary-layer (ABL) flows, and the corresponding surface fluxes of momentum and scalars.

The interaction between the ABL and topography has been the focus of numerous previous studies (e.g., Mason and Sykes 1979; Kustas and Brutsaert 1986; Wood and Mason 1993; Weng et al. 1997; Brown and Wood 2003). The drag force that landscapes exert on the ABL attracts particular attention (e.g., Wood and Mason 1993) because of its significant influence on the ABL flow and turbulent fluxes, which need to be parametrized in atmospheric numerical models. For ABL flow over topography, the drag force consists of both surface friction drag and form drag induced by the topography. There are several ways to account for the overall effect of both types of drag on the flow. These include the bulk similarity approach, consisting of using an effective aerodynamic roughness length within the framework of Monin-Obukhov similarity theory (e.g., Grant and Mason 1990; Wood and Mason 1993; Milton and Wilson 1996; Brown and Wood 2003), and the orographic stress approach (Wood et al. 2001). The effective aerodynamic roughness length approach is the most commonly used. For natural landscapes, due to their multiscale properties, it is reasonable to expect that the effective aerodynamic roughness should depend on the numerical model grid resolution. This potential scale-dependence of the effective aerodynamic roughness should be taken into account in relatively high-resolution models, for which the grid resolution falls within the power-law scaling range of the topography.

Several field studies have been carried out to determine the effective roughness length of natural landscapes (e.g., Kustas and Brutsaert 1986; De Vries et al. 2003). In these studies, the effective roughness is obtained by applying bulk similarity theory to vertical wind-profile data collected using radiosondes (e.g., Kustas and Brutsaert 1986) or meteorological towers (e.g., De Vries et al. 2003). Another way to estimate the effective roughness length of natural landscapes from field measurements is to collect land-surface information via airborne laser altimetry (e.g., De Vries et al. 2003) and apply parametrization schemes (e.g., Kustas and Brutsaert 1986; Grant and Mason 1990) based on surface geometrical parameters such as the frontal or silhouette area of the hills and the base area of the hills.

Most large-eddy simulation (LES) studies of flow over topography have been restricted to flows over simple topographies consisting of two- or three-dimensional idealized shapes (e.g., Wood and Mason 1993; Besio et al. 2000; Brown and Wood 2003; Ding et al. 2003; Iizuka and Kondo 2004; Wan et al. 2007; Wan and Porté-Agel 2011). Several of those studies have used simulation results to determine the effective roughness length of the simple topographies (e.g., Wood and Mason 1993; Besio et al. 2000; Brown and Wood 2003). However, LES has yet to be used to study flow over explicitly-resolved multi-scale topography and to investigate the scaling properties of the corresponding effective surface aerodynamic roughness for different grid resolutions.

In this paper, LES is used to study the effect of multi-scale subgrid-scale (SGS) topography on the overall SGS drag exerted by landscapes on the ABL. It is important to note here 
that the term SGS refers to quantities that are associated with scales smaller than the grid resolution in other numerical models (different from the LES used in this study) that have coarser resolution (e.g., coarser LES models or high-resolution weather forecast models). In particular, the LES results are used to investigate how the effective roughness length relates to surface information such as the SGS variance of surface elevation. To achieve that, a landscape evolution model, the so-called restricted solid-on-solid (RSOS) model (Kim and Kosterlitz 1989), is used to generate a periodic multi-scale topography with statistical properties (e.g., the power spectrum of the elevation field) similar to those of natural landscapes. LESs with the tuning-free scale-dependent Lagrangian dynamic subgrid model (Stoll and Porté-Agel 2006) are performed to simulate ABL flow over multiscale landscapes, and the results used to study the behaviour of the corresponding effective roughness length. Next, brief summaries of the LES technique and the RSOS landscape model are provided.

\subsection{Large-Eddy Simulation}

Large-eddy simulation has been widely used to study the effect of topography on turbulent transport in boundary-layer flow (e.g., Krettenauer and Schumann 1992; Walko et al. 1992; Henn and Sykes 1999; Brown et al. 2001; Iizuka and Kondo 2004; Wan et al. 2007; Wan and Porté-Agel 2011). LES solves the Navier-Stokes equations that have been filtered using a three-dimensional spatial filter. As a result, LES explicitly resolves all the turbulent flow scales larger than the filter size, and parametrizes smaller scales using a subfilter-scale (SFS) model. The filtered Navier-Stokes equations, written in rotational form, are:

$$
\frac{\partial \widetilde{u}_{i}}{\partial t}+\widetilde{u}_{j}\left(\frac{\partial \widetilde{u}_{i}}{\partial x_{j}}-\frac{\partial \widetilde{u}_{j}}{\partial x_{i}}\right)=-\frac{1}{\rho} \frac{\partial \tilde{p}^{*}}{\partial x_{i}}-\frac{\partial \tau_{i j}}{\partial x_{j}}+F_{i},
$$

where $\widetilde{u}_{i}$ is the filtered velocity in the $i$ direction, $\widetilde{p}^{*}=\widetilde{p}+\frac{1}{2} \rho \widetilde{u}_{j} \widetilde{u}_{j}$ is the modified pressure, $\tilde{p}$ is the filtered pressure, $\rho$ is the air density, $\tau_{i j}$ is the SFS stress tensor, and $F_{i}$ is a forcing term (e.g., a mean pressure gradient in the streamwise direction). The SFS stresses $\tau_{i j}$ are parametrized using a SFS model.

An eddy-viscosity model is often used to parametrize the deviatoric part of the SFS stress as follows (Smagorinsky 1963):

$$
\begin{aligned}
\tau_{i j}-\frac{1}{3} \delta_{i j} \tau_{k k} & =-2 v_{\mathrm{sgs}} \widetilde{S}_{i j}, \\
v_{\mathrm{sgs}} & =\left[C_{\mathrm{S}} \Delta\right]^{2}|\widetilde{S}|,
\end{aligned}
$$

where $\widetilde{S}_{i j}=\frac{1}{2}\left(\frac{\partial \widetilde{u}_{i}}{\partial x_{j}}+\frac{\partial \widetilde{u}_{j}}{\partial x_{i}}\right)$ is the resolved strain-rate tensor, $v_{\text {sgs }}$ is the eddy viscosity, the strain-rate tensor magnitude is calculated as $|\widetilde{S}|=\left(2 \widetilde{S}_{i j} \widetilde{S}_{i j}\right)^{\frac{1}{2}}, \Delta$ is the size of the spatial filter, and $C_{\mathrm{S}}$ is the Smagorinsky coefficient.

One of the main challenges in the implementation of the Smagorinsky model is the specification of the model coefficient. The value of $C_{\mathrm{S}}$ is well established for isotropic turbulence. In that case, if a cut-off filter is used in the inertial subrange and the filter scale $\Delta$ is equal to the grid size, then $C_{\mathrm{S}} \approx 0.17$ (Lilly 1967). However, anisotropy of the flow, particularly the presence of a strong mean shear near the surface and around topography in high-Reynoldsnumber ABL flows, makes the optimum value of $C_{\mathrm{S}}$ depart from its isotropic counterpart (e.g., Stoll and Porté-Agel 2006; Wan et al. 2007; Wan and Porté-Agel 2011).

To avoid the need for a priori specification or tuning of the model coefficient, Germano et al. (1991) proposed the so-called dynamic procedure, which calculates the value of the 
model coefficient at every time and position in the flow based on information from the smallest resolved scales. In particular, it minimizes the error incurred when using the Smagorinsky model to compute the resolved Leonard stress (defined using a test filter scale $\bar{\Delta}$, typically of size $2 \Delta$ ) while assuming scale invariance of the coefficient in that range of scales. However, recent studies have shown that the assumption of scale invariance in the dynamic model can lead to errors in simulated boundary-layer flows over both flat surfaces (Porté-Agel et al. 2000) as well as complex terrain (Iizuka and Kondo 2004; Wan et al. 2007; Wan and Porté-Agel 2011).

By relaxing the assumption of scale invariance in the dynamic model, Porté-Agel et al. (2000) proposed the scale-dependent dynamic model. In this model, two test filters (typically of sizes $2 \Delta$ and $4 \Delta$ ) are introduced and used in the dynamic procedure, together with the assumption that the model coefficient has a power-law dependence with scale. When implementing the scale-dependent dynamic model, one needs to use some kind of averaging to guarantee numerical stability. Stoll and Porté-Agel (2006) proposed the Lagrangian scaledependent dynamic model, which employs Lagrangian averaging (Meneveau et al. 1996). Lagrangian averaging consists of averaging over flow pathlines, which makes this model particularly suitable for simulations of non-homogeneous flows. Further details about the Lagrangian scale-dependent dynamic model can be found in Stoll and Porté-Agel (2006).

The performance of the Lagrangian scale-dependent dynamic model has been assessed and compared with that of other commonly used SGS models in simulations of neutral (Wan et al. 2007) as well as stably-stratified (Wan and Porté-Agel 2011) boundary-layer flow over simple sinusoidal hills. By comparing simulation results with wind-tunnel data, these studies showed that the Lagrangian scale-dependent dynamic model, which allows for scale dependence of the eddy-viscosity and eddy-diffusivity model coefficients associated with flow anisotropy, predicts more accurate turbulence statistics than the standard Smagorinsky model and Lagrangian (scale-invariant) dynamic model. For this reason, the Lagrangian scale-dependent dynamic model (Stoll and Porté-Agel 2006) is employed in this study to parametrize SFS stresses in neutral boundary-layer flow over multiscale topography.

\subsection{Landscape Modelling}

In order to perform large-eddy simulations of ABL flow over multiscale topography using periodic boundary conditions compatible with the pseudospectral numerical methods used by many LES models, it is desirable to use surface elevation fields that are periodic. To achieve that, here we generate synthetic periodic multiscale complex terrain using a landscape evolution model, the RSOS model. This model was proposed by Kim and Kosterlitz (1989), and it was initially used to simulate the growth of surface interfaces. Park and Kahng (1995) derived the continuous Kardar-Parisi-Zhang (KPZ) equation (Kardar et al. 1986) from the RSOS model, and proved they are the same in the continuum limit. Both the KPZ equation and the RSOS model have been used extensively to simulate landscape evolution and surface growth (e.g., Kim et al. 2000; Pelletier 2007).

In the RSOS procedure proposed by Kim and Kosterlitz (1989), the surface elevation of a two-dimensional lattice of points is updated by random deposition by using the following rules: the elevation of the point is increased by one if the elevation at this point is smaller than or equal to the elevation of all of its four nearest neighbouring points; however, if this criterion is not satisfied, the elevation of the point remains unchanged. This rule ensures that the elevation difference between any point and its nearest four neighbouring points can only take the value of $-1,0$ or 1 , which prevents the formation of overhangs or vacancies. 
This random deposition process is repeated until a steady-state condition is attained for which the surface height is equal to or greater than the linear dimension of the lattice.

The elevation power spectra of the surfaces generated with the RSOS model show a powerlaw dependence on wavenumber with exponent close to -2 over a wide range of scales. This makes the surface a good approximation to natural landscapes (e.g., Huang and Turcotte 1989). An important advantage of the RSOS model, compared to other synthetic topography generation models that assume a Gaussian probability distribution function (PDF) of topography, is that it is able to produce topographies that have more realistic PDFs. The topography of the earth's surface is skewed in such a way that a much larger percentage of the total landscape is represented by lowlands (topography with an elevation smaller than the median elevation for a region) than highlands. The RSOS model is able to capture this skewness characteristic of the PDF of the elevation of natural landscapes, thus yielding more realistic elevation fields compared with Gaussian models, which are only able to produce a non-skewed Gaussian PDF of elevation (e.g., Weissel et al. 1994; Pelletier 1997).

To understand the effect of multi-scale topography on SGS drag forces that require parametrization in high-resolution numerical models (e.g., weather forecast models or LES), it is of interest to study the relationship between effective aerodynamic roughness and SGS landscape characteristics. In particular, the aerodynamic roughness is expected to increase with increasing SGS variance of the elevation field (variance of surface elevation associated with the SGS topography). The SGS variance of the elevation field is defined as:

$$
\sigma_{\mathrm{h}, \mathrm{sgs}}^{2}=\langle\widehat{h h}-\widehat{h h}\rangle,
$$

where $h$ is the surface elevation, the hat symbol and $\langle\quad\rangle$ denote spatial filtering at a resolution of the numerical model $\left(\Delta_{\text {model }}\right)$ and area averaging, respectively. Note that, in the case of zero SGS variance (no SGS topography), the effective roughness is equal to the aerodynamic roughness of the surface. Besides this limiting behaviour, the functional relationship between effective roughness and SGS variance of elevation is not known. As a first-order approximation, one could assume a linear relation between the effective roughness and the variance of the elevation field (e.g., Zagarola and Smits 1998; Flack and Schultz 2010; Anderson and Meneveau 2011). Furthermore, in order to comply with the above-mentioned lower limit for the aerodynamic roughness, here we propose to use:

$$
\left(z_{\mathrm{o}}^{\mathrm{eff}}\right)^{2}-z_{\mathrm{o}}^{2}=C \sigma_{\mathrm{h}, \mathrm{sgs}}^{2}=C\langle\widehat{h h}-\widehat{h} \widehat{h}\rangle,
$$

where $C$ is a proportionality factor, assumed to be constant for a given landscape (topography and aerodynamic roughness combination).

In our study, large-eddy simulations using the scale-dependent Lagrangian dynamic model are performed of ABL flows over a series of multiscale complex topographies. The simulation results are used to examine the relation between SGS variance of elevation $\sigma_{\mathrm{h}, \mathrm{sgs}}^{2}$ and effective roughness length associated with the SGS topography. Particular emphasis is placed on evaluating the linear model given by Eq. (5). Simulation details are explained in the following section.

\section{Numerical Experiments}

A detailed description of the LES code used in this numerical study can be found in Albertson and Parlange (1999), Porté-Agel et al. (2000), Stoll and Porté-Agel (2006) and Wan et al. (2007). The spatial derivatives are computed using pseudospectral methods in the horizontal directions with periodic boundary conditions, and finite differences in the vertical direction. 
Fig. 1 Multiscale complex terrain $T_{0}$ obtained using the RSOS model after $8 \times 10^{6}$ times. All dimensions have been normalized with a length scale of $L=1000 \mathrm{~m}$

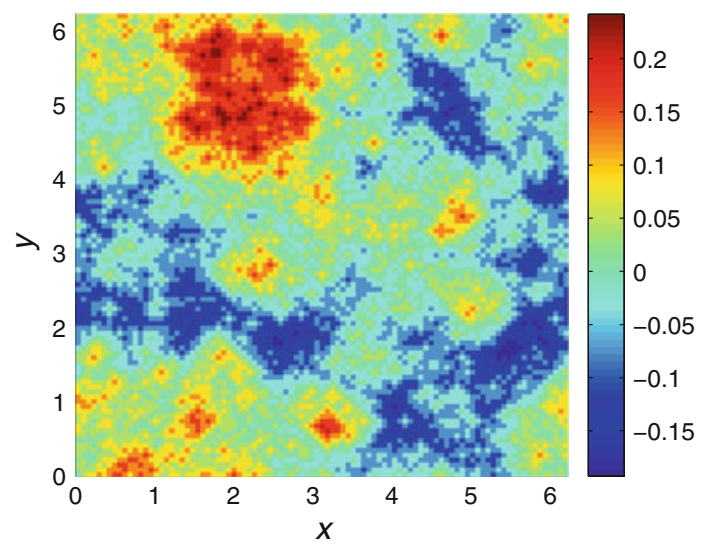

Fig. 2 Elevation power spectral density of multiscale complex terrain $T_{0}$ against wavenumber (solid line). The dash-dot line show the -1.8 slope

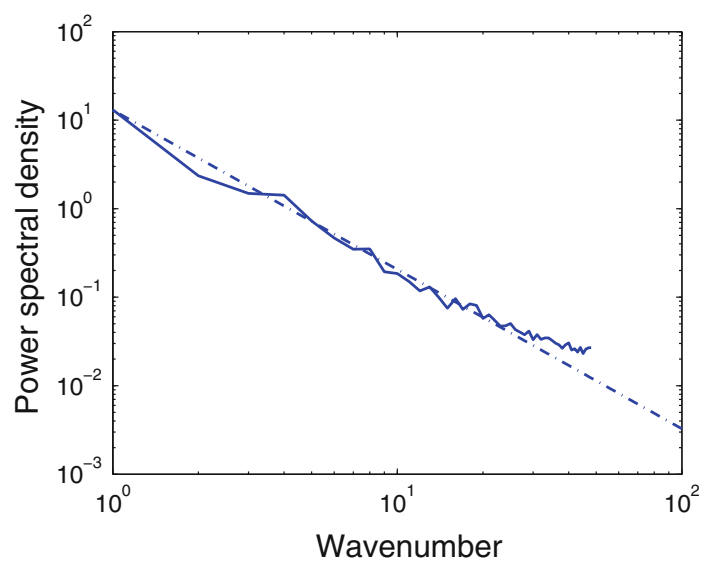

The lower boundary condition applies similarity theory (the surface-layer logarithmic law) to calculate the instantaneous (filtered) surface shear stress based on the velocity field at the lowest computational level. The upper boundary condition is a fixed stress-free lid, while time advancement is realized through the second-order Adams-Bashforth scheme.

The lower surface of the physical domain consists of a series of periodic multiscale topographies obtained by spatially filtering a multiscale terrain generated using the RSOS model (Kim and Kosterlitz 1989). These terrains approximate (statistically) SGS topography of natural landscapes. The RSOS model is applied here starting from a flat initial surface of zero elevation, and discretizing it as a lattice with $96 \times 96$ uniformly distributed grid points. Periodic boundary conditions are enforced in both horizontal directions. The RSOS procedure described above is applied $8 \times 10^{6}$ times to obtain a multiscale complex terrain (not shown here). The simulated terrain is periodic in both $x$ and $y$ directions, and is then shifted to a mean height of zero and scaled in all three directions to fit the LES domain dimensions, which have non-dimensional values of $2 \pi, 2 \pi$ and 2 . A surface $T_{0}$ (shown in Fig. 1) is therefore obtained. The elevation power spectral density of terrain $T_{0}$ is plotted against wavenumber in a $\log -\log$ scale in Fig. 2. The spectral density of elevation shows a clear -1.8 slope, which is very close to the -2 slope of the elevation power spectrum reported for natural topography (e.g., Huang and Turcotte 1989); this feature makes the obtained terrain 
Table 1 The topographic statistics of lower surfaces of the LES physical domain

\begin{tabular}{llllll}
\hline SGS terrain & Scales & $\begin{array}{l}\text { SGS variance } \\
\text { of elevation }\end{array}$ & $\begin{array}{l}\text { Maximum } \\
\text { height }\end{array}$ & $\begin{array}{l}\text { Minimum } \\
\text { height }\end{array}$ & $\begin{array}{l}\text { Maximum } \\
\text { slope }\end{array}$ \\
\hline Flat & $4 \sqrt{2} \Delta$ to $4 \sqrt{2} \Delta$ & 0 & 0 & 0 & 0 \\
$T_{1}$ & $4 \sqrt{2} \Delta$ to $8 \Delta$ & $2.46 \times 10^{-4}$ & 0.0517 & -0.0486 & 0.2539 \\
$T_{2}$ & $4 \sqrt{2} \Delta$ to $8 \sqrt{2} \Delta$ & $9.99 \times 10^{-4}$ & 0.1126 & -0.0894 & 0.4510 \\
$T_{3}$ & $4 \sqrt{2} \Delta$ to $16 \Delta$ & $1.44 \times 10^{-3}$ & 0.1417 & -0.1241 & 0.4983 \\
$T_{4}$ & $4 \sqrt{2} \Delta$ to $16 \sqrt{2} \Delta$ & $1.91 \times 10^{-3}$ & 0.1587 & -0.1331 & 0.5161 \\
$T_{5}$ & $4 \sqrt{2} \Delta$ to $32 \sqrt{2} \Delta$ & $5.36 \times 10^{-3}$ & 0.2022 & -0.1779 & 0.5603 \\
\hline
\end{tabular}

a reasonable approximation to natural landscapes for the purpose of studying the effect of multiscale topography on ABL flow.

Starting from the RSOS-simulated multiscale terrain shown in Fig. 1, we generate a series of multiscale complex terrains containing different ranges of scales in the elevation power spectrum. This is achieved by means of two-dimensional band-pass spatial filtering. The resulting landscapes can be considered to be SGS topography in larger-scale models (e.g., LES or weather forecast models) of different spatial resolutions. In order to guarantee that the effects of all scales existing in the considered terrains are properly captured by the LES, first we apply a low-pass filter at scale $4 \sqrt{2} \Delta$ (about six times the LES grid size $\Delta$ ). This filtering operation retains only the terrain fluctuations of horizontal size larger than that scale. Therefore, that scale can be considered as the smallest size of topographic features that can be identified in the simulated terrain (corresponding to the scale below which hill-slope diffusion processes dominate during landscape evolution). Furthermore, in order to simulate the effect of different ranges of scales (SGS in larger-scale models), we apply a second high-pass filter at different scales: $4 \sqrt{2} \Delta, 8 \Delta, 8 \sqrt{2} \Delta, 16 \Delta, 16 \sqrt{2} \Delta$, and $32 \sqrt{2} \Delta$, respectively, and obtain six filtered terrains. It is important to point out that, at scale $32 \sqrt{2} \Delta$, the filtered terrain contains all the large scales of the original RSOS terrain. Considering elevation fields between scale $4 \sqrt{2} \Delta$ and $8 \Delta, 4 \sqrt{2} \Delta$ and $8 \sqrt{2} \Delta, 4 \sqrt{2} \Delta$ and $16 \Delta, 4 \sqrt{2} \Delta$ and $16 \sqrt{2} \Delta, 4 \sqrt{2} \Delta$ and $32 \sqrt{2} \Delta$, we obtain a series of SGS terrains: $T_{1}, T_{2}, T_{3}, T_{4}, T_{5}$ (shown in Fig. 3). From Fig. 3, it is clear that, as the scale increases, the magnitude of terrain elevation increases, and largersize topographic structures are included in the terrains. The statistics of the SGS terrains considered here are shown in Table 1. As expected, the inclusion of more (larger) scales in the terrains from $T_{1}$ to $T_{5}$ leads to an increase in the level of surface variability, which results in increasing SGS variance of elevation.

The LES models uses the terrain-following coordinate transformation developed by Clark (1977). It is used to transform the physical domains containing the multiscale topographies $T_{1}$ to $T_{5}$ into a flat computational domain by using the following equation:

$$
\bar{z}=H\left(\frac{z-z_{\mathrm{s}}(x, y)}{H-z_{\mathrm{s}}(x, y)}\right),
$$

where $\bar{z}$ is the vertical position in the transformed system, $z$ represents distance to the complex surface in the original system, $z_{\mathrm{s}}$ and $H$ denote the actual elevation (in the original system) of the terrain and the top of the domain, respectively.

The dimensions of the computational domain are $2 \pi, 2 \pi$ and 2 in the streamwise, spanwise and vertical directions, respectively, after being normalized with a length scale of $L=$ $1000 \mathrm{~m}$. Three different non-dimensional surface roughness lengths are used in the simula- 

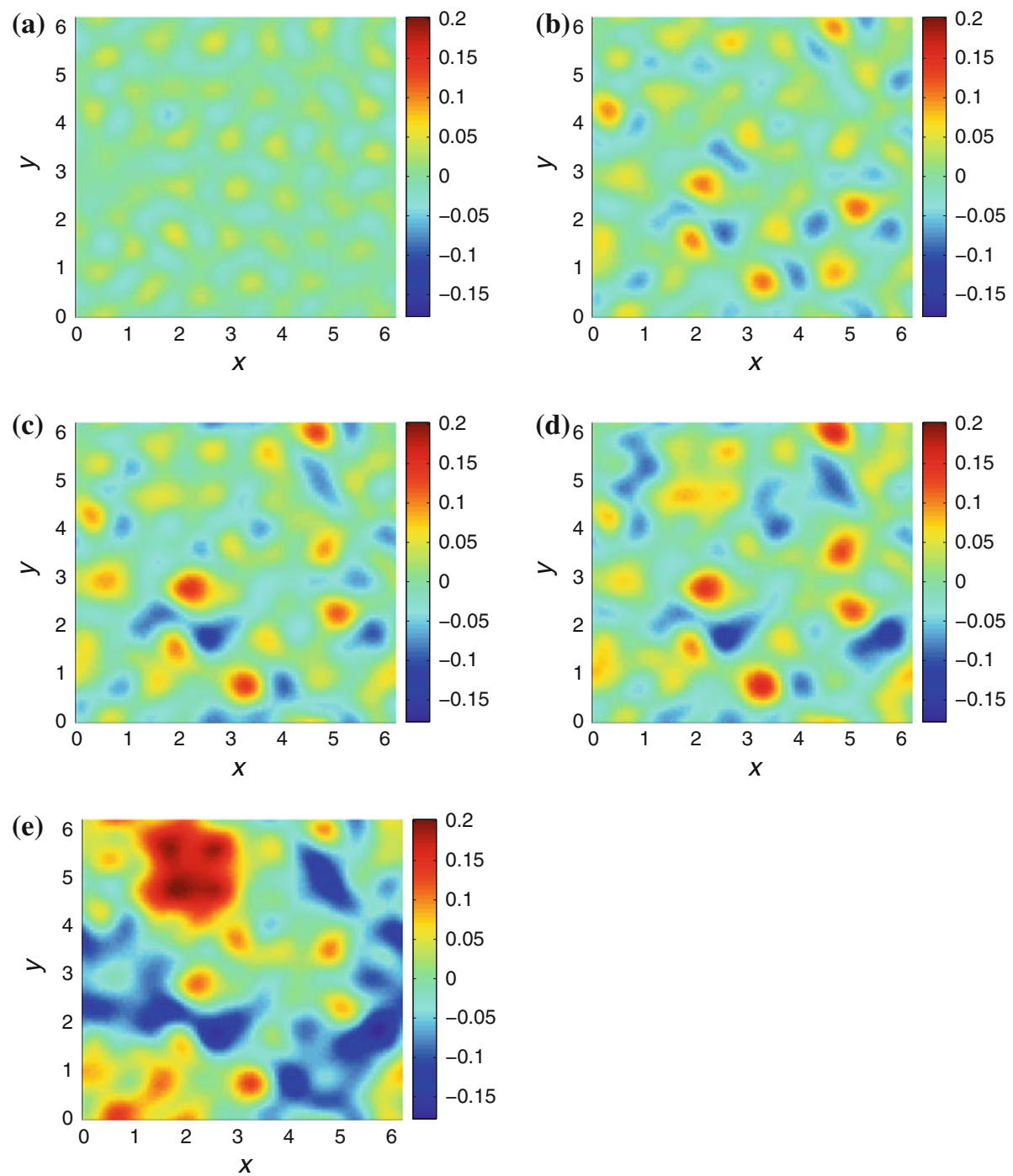

Fig. 3 SGS terrains used as lower surfaces of the physical domain in LES: a $T_{1}$, b $T_{2}$, c $T_{3}$, d $T_{4}$, e $T_{5}$. All dimensions have been normalized with a length scale of $L=1000 \mathrm{~m}$

tions, with values set at $10^{-4}, 2 \times 10^{-4}$ and $5 \times 10^{-4}$, respectively. The computational domain is divided into $96 \times 96 \times 128$ uniformly spaced grid points; this yields a grid aspect ratio of $4: 4: 3 / \pi$. The grid is staggered in the vertical direction, with the vertical velocity stored halfway between the other variables. The flow is driven by a constant pressure gradient in the streamwise direction with a non-dimensional magnitude of 0.5 , while the velocity scale $u_{\mathrm{o}}$ used for normalization is taken to be $0.45 \mathrm{~m} \mathrm{~s}^{-1}$.

Large-eddy simulations of ABL flow are performed over the terrains shown in Fig. 3 and described in Table 1 (including a flat surface). Simulations are run for a period of time long enough to guarantee statistical convergence of the results; the duration of the simulations 
is $2.67 \times 10^{5} \mathrm{~s}$. The averaging period used in the simulations is the last $7.2 \times 10^{4} \mathrm{~s}$ of the simulation duration, with flow statistics presented and analyzed in the following section.

\section{Results}

Previous numerical (Newley 1985; Wood and Mason 1993; Besio et al. 2000; Wood and Mason 1993) and experimental (Thompson 1978; Kustas and Brutsaert 1986; Grant and Mason 1990) studies of flow over hills have shown that the area-averaged streamwise velocity varies logarithmically with height in the surface layer. Unlike the standard logarithmic law for neutral flow over homogeneous flat surfaces, which is based on the actual friction velocity $\left(u_{*}\right)$ and the aerodynamic roughness of the surface $\left(z_{0}\right)$, the logarithmic law for areaaveraged velocity over hilly surfaces is formulated using an effective roughness length and an effective friction velocity. Therefore, the area-averaged velocity over complex topography can be written as

$$
\langle u\rangle=\frac{u_{*}^{\text {eff }}}{\kappa} \ln \left(\frac{z-d}{z_{\mathrm{o}}^{\text {eff }}}\right),
$$

where $\langle u\rangle$ is the area-averaged velocity over horizontal planes, $u_{*}^{\text {eff }}$ is the effective friction velocity, $\kappa$ is the von Karman constant, $d$ is the effective displacement height, and $z_{0}^{\text {eff }}$ is the effective roughness length. The effective friction velocity is equal to the square root of the total kinematic surface force (shear stress plus drag form) per unit area.

Previous studies (e.g., Wood and Mason 1993) suggest that the effective displacement height is equal to the mean height of surface elevation for hilly terrains. In the present study, all surfaces under consideration have a mean height of zero, making it reasonable for us to choose an effective displacement height of zero. Due to the constant pressure gradient exerted on the flow, the normalized simulated area-averaged total turbulent stress changes linearly with height, from a value of -1 at the surface to a value of zero at the top of the boundary layer. Consistent with that, the non-dimensional effective friction velocity $u_{*}^{\text {eff }}$ has a value of 1.0 for all the simulations. Taking advantage of the known effective friction velocity $u_{*}^{\text {eff }}$ as well as the area-averaged velocity $\langle u\rangle$ obtained from the simulations, and applying Eq. 7, we are able to determine the effective roughness length for all the different terrains under consideration.

The area-averaged non-dimensional velocity profiles from the simulations over the series of terrains considered here, with a non-dimensional surface roughness length of $z_{\mathrm{o}}=10^{-4}$, are shown in Fig. 4a-f with symbols. From those figures, we can see that all profiles show a logarithmic dependence with height in the surface layer, i.e., the lowest $15 \%$ of the domain. Knowing that the effective friction velocity $u_{*}^{\text {eff }}$ is the same for all simulations, we can apply least squares fitting to the area-averaged velocity in the logarithmic region (Besio et al. 2000; Athanassiadou and Castro 2001) with straight lines of a constant slope of $2.5\left(=u_{*}^{\text {eff }} / \kappa\right)$. The effective roughness length can then be obtained by finding the intercept of the straight fitted lines with the $y$-axis, where the average velocity is zero. The fitted lines are shown as solid lines in Fig. 4 together with the coefficients of determination $\left(R^{2}\right)$ obtained in the fitting. The SGS variance of elevation $\sigma_{\mathrm{h}, \mathrm{sgs}}^{2}$, surface roughness length $z_{\mathrm{o}}$, computed effective roughness length $z_{\mathrm{o}}^{\text {eff }}$, and the quantity $\left(z_{\mathrm{o}}^{\text {eff }}\right)^{2}-z_{\mathrm{o}}^{2}$ are listed in Table 2 for all terrains under consideration.

Figures 5 and 6 show the area-averaged non-dimensional velocity profiles from simulations over the same series of SGS terrains, but now with non-dimensional surface roughness 

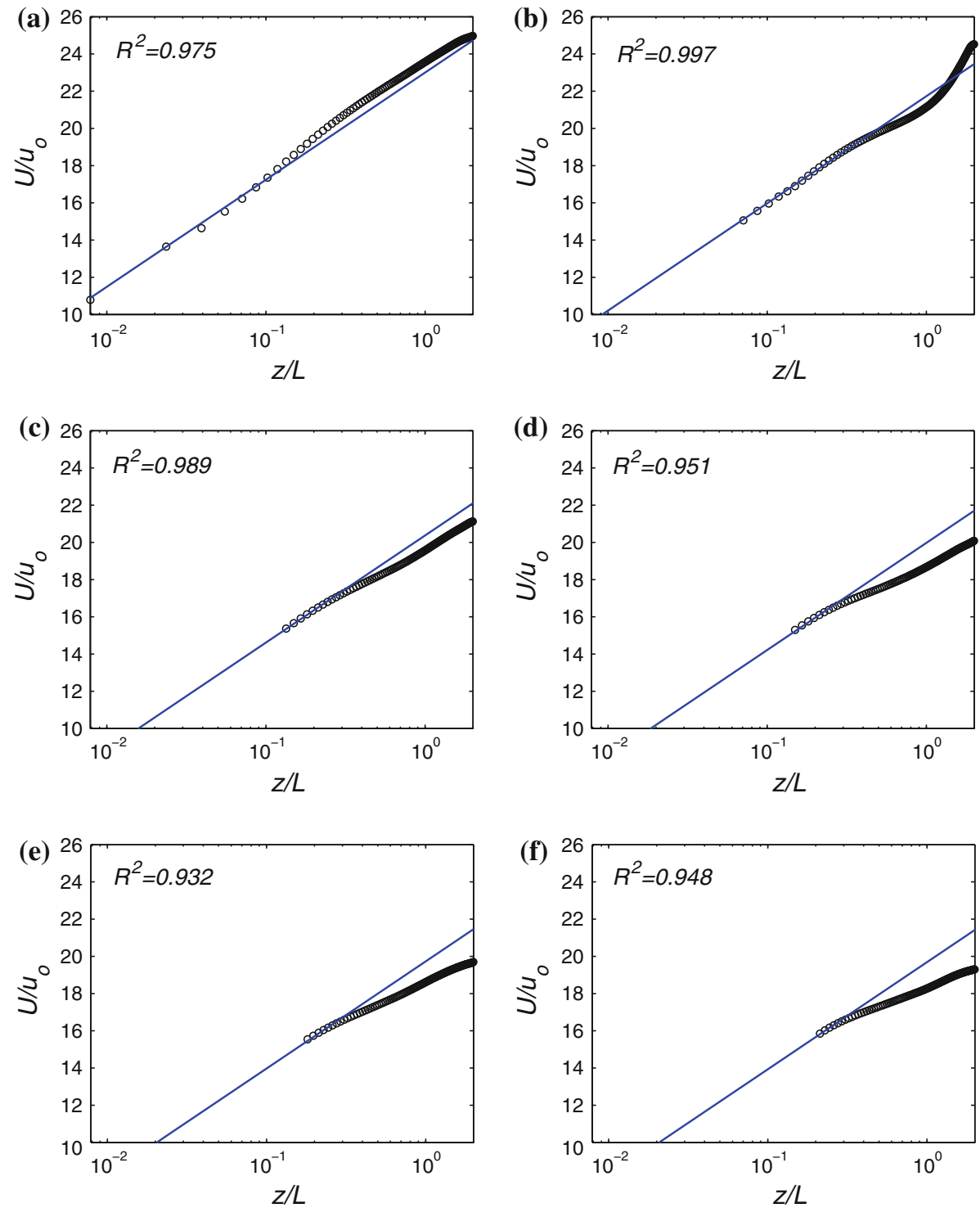

Fig. 4 Area-averaged non-dimensional velocity profiles (shown in symbols) against height for the SGS terrains: a flat terrain, b $T_{1}$, c $T_{2}, \mathbf{d} T_{3}$, e $T_{4}$, f $T_{5}$. Least squares fitted lines for the lowest $15 \%$ part are also shown (solid lines). Non-dimensional surface roughness length for all the terrains is $10^{-4}$. The coefficient of determination $\left(R^{2}\right)$ from the linear regression in the surface layer is shown in all figures

lengths of $2 \times 10^{-4}$ and $5 \times 10^{-4}$, respectively. Again, in the lowest $10-15 \%$ of the domain, the velocity increases as the logarithm of height for all the simulations. Using the same least squares fitting method described above, the effective roughness lengths for the SGS terrains can be calculated. The obtained effective roughness values are shown in Table 2. From Figs. 4, 5 and 6, we can see that the wind speed appears to gradually decrease with increasing orographic variance. Since the horizontal pressure gradient forcing is the same for all 
Table 2 SGS variance of elevation, surface roughness length, effective roughness length and an introduced quantity $\left(z_{\mathrm{O}}^{\mathrm{eff}}\right)^{2}-z_{\mathrm{O}}^{2}$ for all the terrains under consideration

\begin{tabular}{llllll}
\hline SGS terrain & Scales & $\begin{array}{l}\text { SGS variance of } \\
\text { elevation }\end{array}$ & $\begin{array}{l}\text { Surface roughness } \\
\text { length }\end{array}$ & $\begin{array}{l}\text { Effective } \\
\text { roughness length }\end{array}$ & $\left(z_{\mathrm{o}}^{\text {eff }}\right)^{2}-z_{\mathrm{o}}^{2}$ \\
\hline Flat & $4 \sqrt{2} \Delta$ to $4 \sqrt{2} \Delta$ & 0 & $1.00 \times 10^{-4}$ & $1.00 \times 10^{-4}$ & 0 \\
$T_{1}$ & $4 \sqrt{2} \Delta$ to $8 \Delta$ & $2.46 \times 10^{-4}$ & $1.00 \times 10^{-4}$ & $1.75 \times 10^{-4}$ & $2.08 \times 10^{-8}$ \\
$T_{2}$ & $4 \sqrt{2} \Delta$ to $8 \sqrt{2} \Delta$ & $9.99 \times 10^{-4}$ & $1.00 \times 10^{-4}$ & $2.88 \times 10^{-4}$ & $7.28 \times 10^{-8}$ \\
$T_{3}$ & $4 \sqrt{2} \Delta$ to $16 \Delta$ & $1.44 \times 10^{-3}$ & $1.00 \times 10^{-4}$ & $3.37 \times 10^{-4}$ & $1.03 \times 10^{-7}$ \\
$T_{4}$ & $4 \sqrt{2} \Delta$ to $16 \sqrt{2} \Delta$ & $1.91 \times 10^{-3}$ & $1.00 \times 10^{-4}$ & $3.69 \times 10^{-4}$ & $1.26 \times 10^{-7}$ \\
$T_{5}$ & $4 \sqrt{2} \Delta$ to $32 \sqrt{2} \Delta$ & $5.36 \times 10^{-3}$ & $1.00 \times 10^{-4}$ & $3.77 \times 10^{-4}$ & $1.32 \times 10^{-7}$ \\
Flat & $4 \sqrt{2} \Delta$ to $4 \sqrt{2} \Delta$ & 0 & $2.00 \times 10^{-4}$ & $2.20 \times 10^{-4}$ & 0 \\
$T_{1}$ & $4 \sqrt{2} \Delta$ to $8 \Delta$ & $2.46 \times 10^{-4}$ & $2.00 \times 10^{-4}$ & $3.03 \times 10^{-4}$ & $5.21 \times 10^{-8}$ \\
$T_{2}$ & $4 \sqrt{2} \Delta$ to $8 \sqrt{2} \Delta$ & $9.99 \times 10^{-4}$ & $2.00 \times 10^{-4}$ & $5.16 \times 10^{-4}$ & $2.26 \times 10^{-7}$ \\
$T_{3}$ & $4 \sqrt{2} \Delta$ to $16 \Delta$ & $1.44 \times 10^{-3}$ & $2.00 \times 10^{-4}$ & $6.37 \times 10^{-4}$ & $3.66 \times 10^{-7}$ \\
$T_{4}$ & $4 \sqrt{2} \Delta$ to $16 \sqrt{2} \Delta$ & $1.91 \times 10^{-3}$ & $2.00 \times 10^{-4}$ & $6.82 \times 10^{-4}$ & $4.25 \times 10^{-7}$ \\
$T_{5}$ & $4 \sqrt{2} \Delta$ to $32 \sqrt{2} \Delta$ & $5.36 \times 10^{-3}$ & $2.00 \times 10^{-4}$ & $6.89 \times 10^{-4}$ & $4.35 \times 10^{-7}$ \\
Flat & $4 \sqrt{2} \Delta$ to $4 \sqrt{2} \Delta$ & 0 & $5.00 \times 10^{-4}$ & $5.00 \times 10^{-4}$ & 0 \\
$T_{1}$ & $4 \sqrt{2} \Delta$ to $8 \Delta$ & $2.46 \times 10^{-4}$ & $5.00 \times 10^{-4}$ & $7.19 \times 10^{-4}$ & $2.66 \times 10^{-7}$ \\
$T_{2}$ & $4 \sqrt{2} \Delta$ to $8 \sqrt{2} \Delta$ & $9.99 \times 10^{-4}$ & $5.00 \times 10^{-4}$ & $1.10 \times 10^{-3}$ & $9.60 \times 10^{-7}$ \\
$T_{3}$ & $4 \sqrt{2} \Delta$ to $16 \Delta$ & $1.44 \times 10^{-3}$ & $5.00 \times 10^{-4}$ & $1.30 \times 10^{-3}$ & $1.44 \times 10^{-6}$ \\
$T_{4}$ & $4 \sqrt{2} \Delta$ to $16 \sqrt{2} \Delta$ & $1.91 \times 10^{-3}$ & $5.00 \times 10^{-4}$ & $1.40 \times 10^{-3}$ & $1.71 \times 10^{-6}$ \\
$T_{5}$ & $4 \sqrt{2} \Delta$ to $32 \sqrt{2} \Delta$ & $5.36 \times 10^{-3}$ & $5.00 \times 10^{-4}$ & $1.50 \times 10^{-3}$ & $2.00 \times 10^{-6}$ \\
\hline
\end{tabular}

simulations, the area-averaged streamwise velocity increases against the logarithm of height at the same rate. For topography with larger orographic variance, however, the near-surface area-averaged streamwise velocity (at the same height) is smaller due to the larger total surface stress. This leads to a relatively smaller wind speed throughout the simulation domain. In order to illustrate the flow field generated by LES, we present in Fig. 7 two sectional cuts of the streamwise velocity field over terrain $T_{5}$ with a non-dimensional surface roughness of $10^{-4}$. Figure 7a shows the time-averaged streamwise velocity field at the central vertical $x z$ plane of the domain, and Fig. $7 \mathrm{~b}$ presents the instantaneous streamwise velocity field at the same plane.

Next, we explore the relationship between the effective aerodynamic roughness and the SGS variance of the surface elevation. In particular, in order to evaluate the validity of the simple linear model given by Eq. (5), the quantity $\left(z_{0}^{\text {eff }}\right)^{2}-z_{0}^{2}$ is plotted in Fig. 8 against SGS variance of elevation $\sigma_{\mathrm{h}, \mathrm{sgs}}^{2}$ for the series of SGS terrains with the three different surface roughness lengths. From Fig. 8, we can conclude that the linear relation between $\left(z_{\mathrm{o}}^{\text {eff }}\right)^{2}-z_{\mathrm{o}}^{2}$ and $\sigma_{\mathrm{h}, \mathrm{sgs}}^{2}$ holds true for most of the scales in all three cases. As mentioned above, it is reasonable that the increasing variance of surface elevation $\left(\sigma_{\mathrm{h}, \mathrm{sgs}}^{2}\right)$ leads to an increase in the effective roughness length. For the three different surface roughness cases considered, that rate of increase (parameter $C$ in Eq. 5) is shown to be constant over a wide range of scales. Only at the largest scale $\left(16 \sqrt{2} \Delta\right.$ in this study) does $\left(z_{0}^{\text {eff }}\right)^{2}-z_{0}^{2}$ no longer increase against $\sigma_{\mathrm{h}, \mathrm{sgs}}^{2}$ at the same rate as at smaller scales. Instead, our results show that those largest 

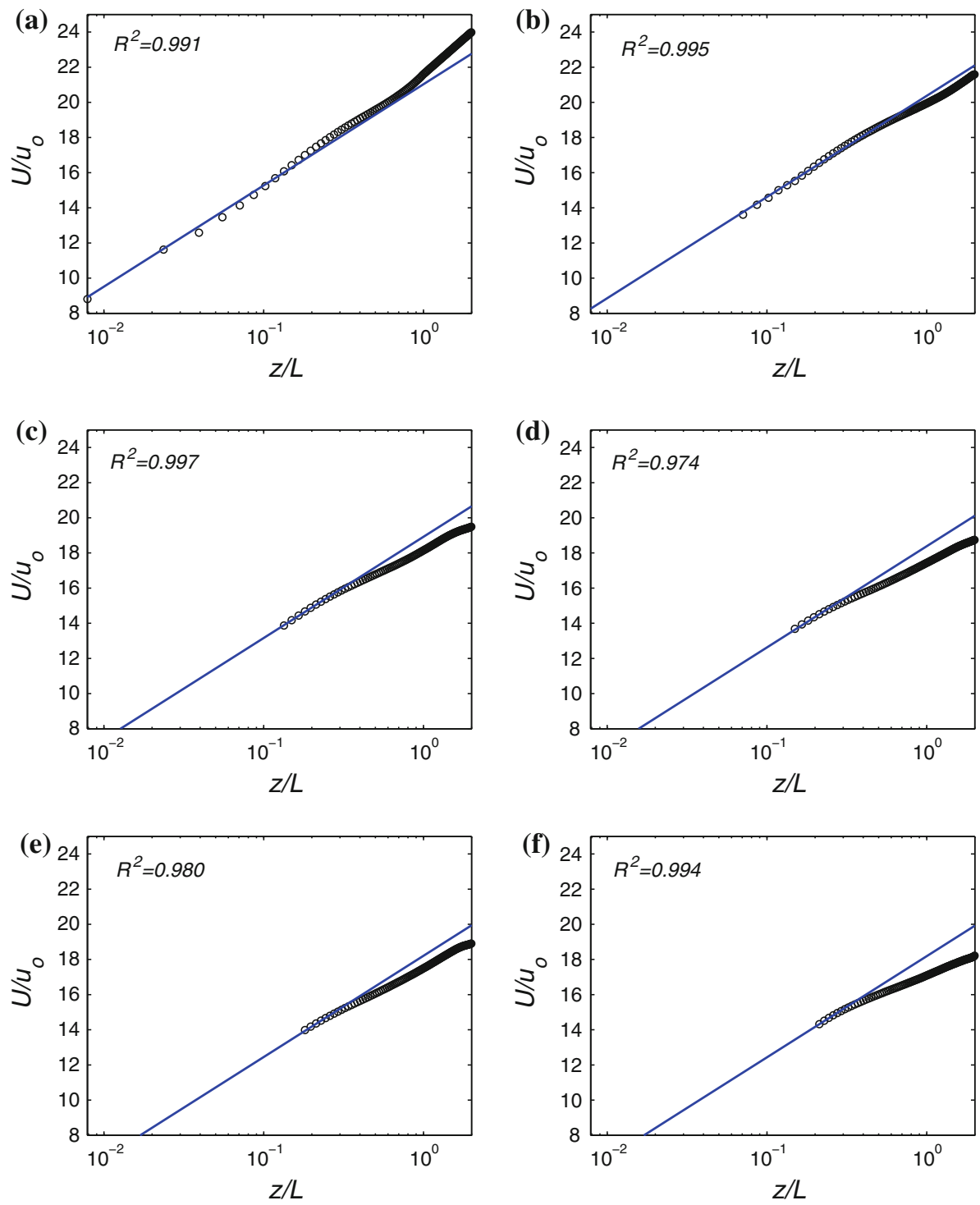

Fig. 5 Area-averaged non-dimensional velocity profiles (shown in symbols) against height for the SGS terrains: a flat terrain, b $T_{1}$, c $T_{2}, \mathbf{d} T_{3}$, e $T_{4}$, f $T_{5}$. Least squares fitted lines for the lowest $15 \%$ part are also shown (solid lines). Non-dimensional surface roughness length for all the terrains is $2 \times 10^{-4}$. The coefficient of determination $\left(R^{2}\right)$ from the linear regression in the surface layer is shown in all figures

scales have practically no effect on the value of the effective roughness. In order to better understand the marginal effect of the largest scales, Fig. 9 shows the elevation field obtained by low-pass filtering the initial multiscale terrain $T_{0}$ (Fig. 1) with a filter of size $16 \sqrt{2} \Delta$. The resulting filtered terrain is very mild and it is characterized by a maximum slope of 6 degrees. The fact that this range of scales (characterized by very mild slope) has little effect on the effective roughness $z_{\mathrm{o}}^{\text {eff }}$ of the surface is consistent with the simulation results of 

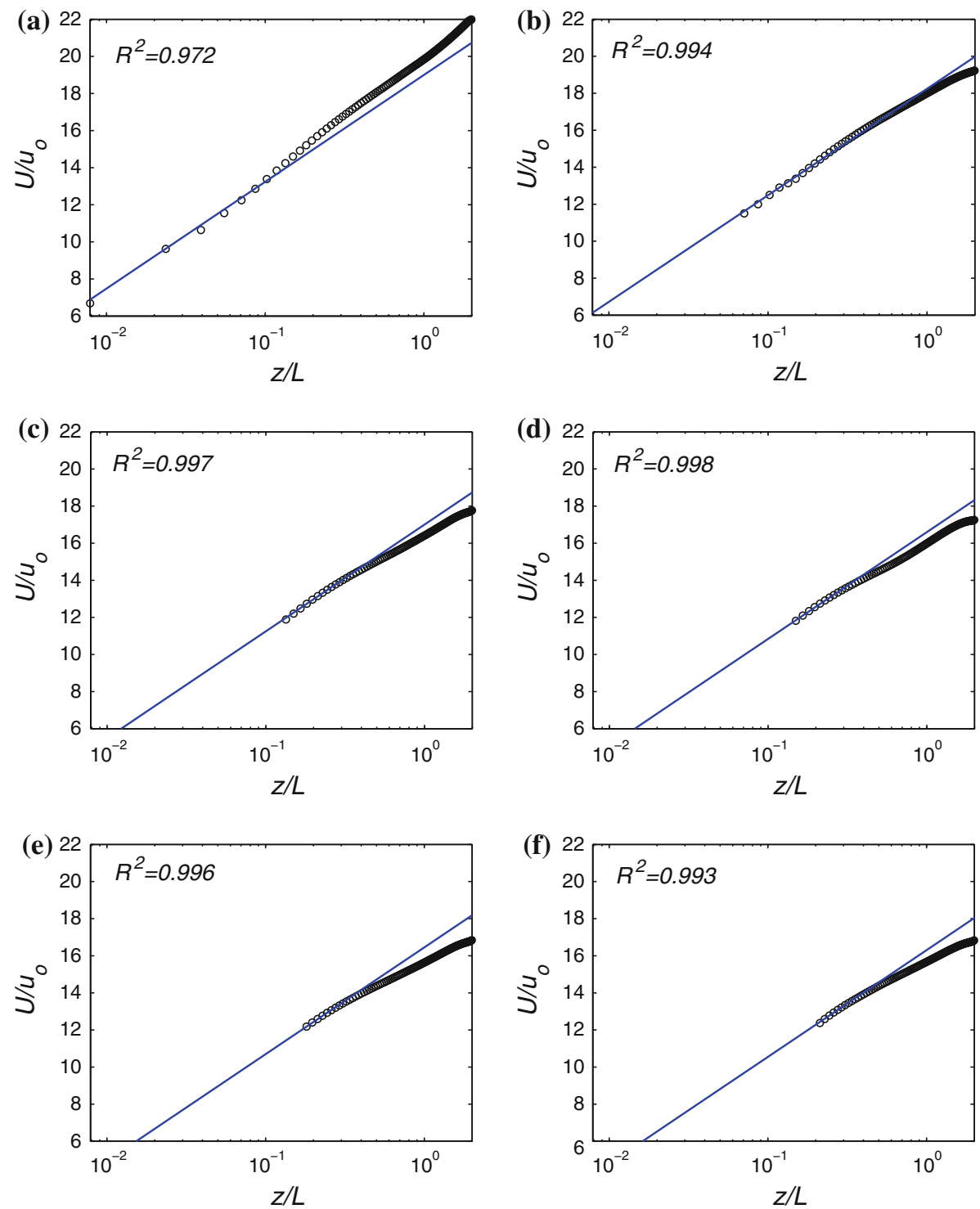

Fig. 6 Area-averaged non-dimensional velocity profiles (shown in symbols) against height for the SGS terrains: a Flat terrain, b $T_{1}$, c $T_{2}$, d $T_{3}$, e $T_{4}$, f $T_{5}$. Least squares fitted lines for the lowest $15 \%$ part are also shown (solid lines). Non-dimensional surface roughness length for all the terrains is $5 \times 10^{-4}$. The coefficient of determination $\left(R^{2}\right)$ from the linear regression in the surface layer is shown in all figures

Wood and Mason (1993). They showed that, in simulations of flow over multiple 'packed' hills of the same size, the effect of the topography on the effective roughness height is very small (less than $20 \%$ increase with respect to the surface roughness) when the maximum slope of the terrain was smaller or equal to 6 degrees (see Table 1 in Wood and Mason 1993).

In order to quantify the value of the parameter $C$ in Eq. (5), i.e., the slope of the linear relation between $\left(z_{\mathrm{o}}^{\mathrm{eff}}\right)^{2}-z_{\mathrm{o}}^{2}$ and $\sigma_{\mathrm{h}, \mathrm{sgs}}^{2}$ for the series of terrains considered here, we apply 

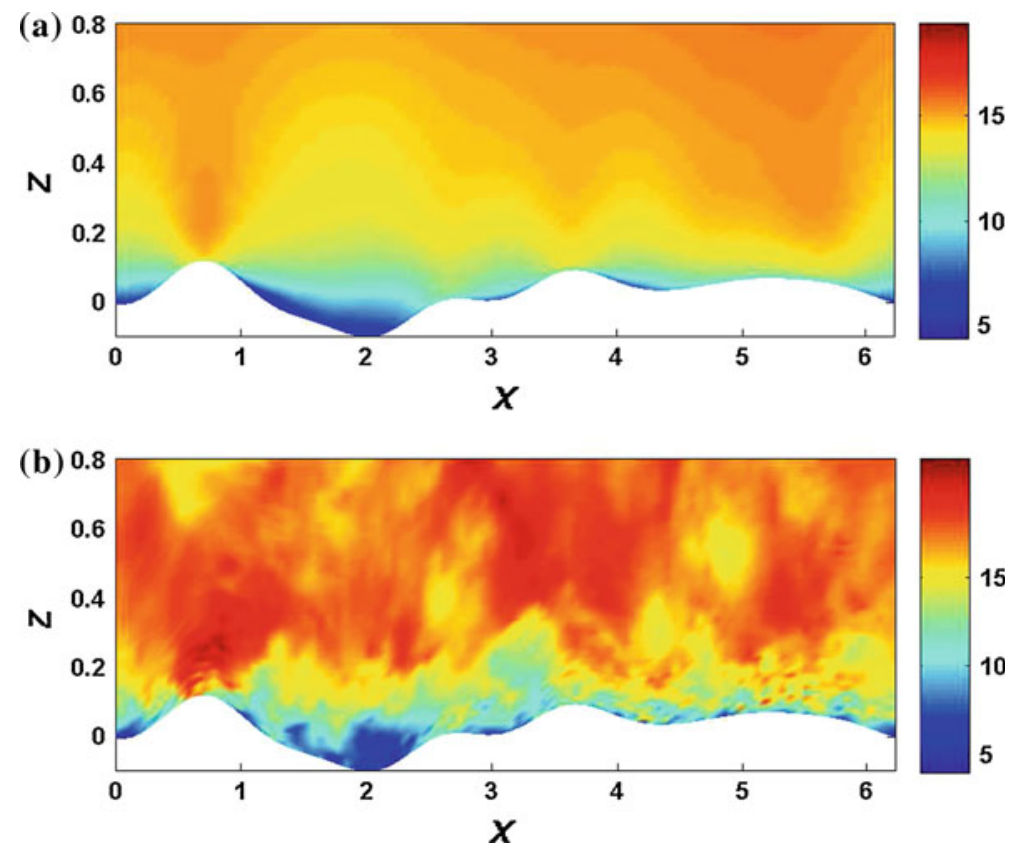

Fig. 7 Non-dimensional streamwise velocity field generated by the LES over $T_{5}$ with a non-dimensional surface roughness of $10^{-4}$ : a time-averaged streamwise velocity field at the central vertical $x z$ plane of the domain, b instantaneous streamwise velocity field at the same plane

Fig. $8\left(z_{\mathrm{o}}^{\text {eff }}\right)^{2}-z_{\mathrm{o}}^{2}$ versus $\sigma_{\mathrm{h}, \mathrm{sgs}}^{2}$ for the series of SGS terrains (shown in symbols): the squares denote results from SGS terrains with a non-dimensional surface roughness length of $10^{-4}$, the circles denote results from SGS terrains with a non-dimensional surface roughness length of $2 \times 10^{-4}$, and the triangles denote results from SGS terrains with a non-dimensional surface roughness length of $5 \times 10^{-4}$.

The solid lines are least squares fitted lines for the linear region

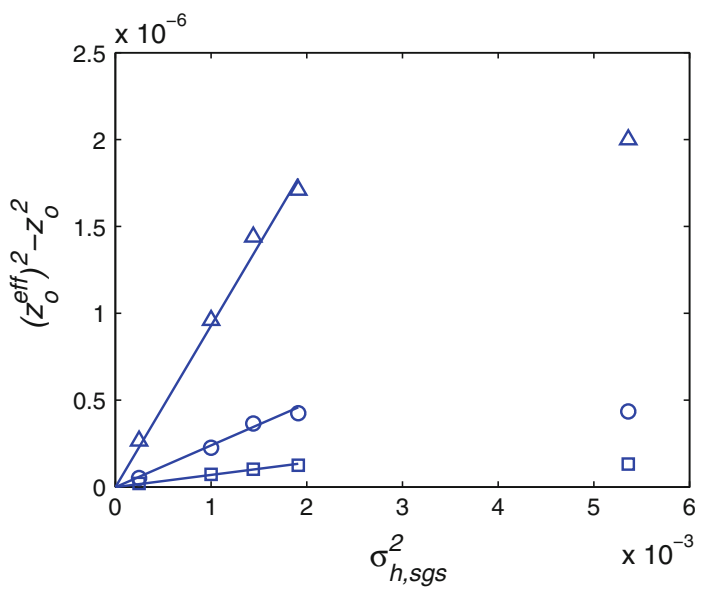

least squares fitting to the points within the linear scaling regions in Fig. 8, and find the corresponding slope. The value of this slope is found to be $7 \times 10^{-5}, 2 \times 10^{-4}$ and $9 \times 10^{-4}$ for the terrains with surface aerodynamic roughness $z_{0}$ of $10^{-4}, 2 \times 10^{-4}$ and $5 \times 10^{-4}$, respectively. It is therefore clear that the slope of the linear relation between $\left(z_{0}^{\text {eff }}\right)^{2}-z_{0}^{2}$ and $\sigma_{\mathrm{h}, \mathrm{sgs}}^{2}$ increases with increasing aerodynamic surface roughness length, a result that is supported by previous wind-tunnel studies performed over simple topographies. In particular, Gong et al. (1996) showed that the two-dimensional sinusoidal hill with a relatively rough 
Fig. 9 Elevation field above scale $16 \sqrt{2} \Delta$ obtained from $T_{0}$

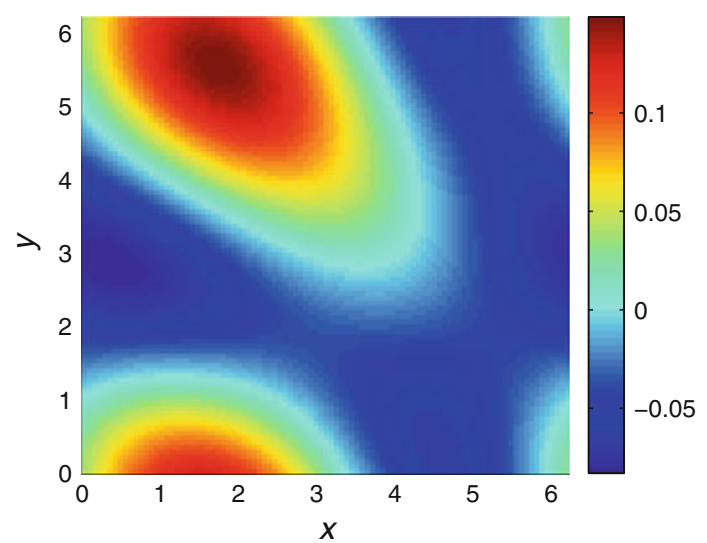

surface yielded a larger total drag (including both friction drag and form drag) than the same hill with a relatively smooth surface (see Table 2 in Gong et al. 1996). This indicates that, for a given topography, a larger surface roughness results in a larger total surface drag as well as a larger effective roughness length.

In summary, our results confirm that the simple linear model given by Eq. 5 can be used to provide a reasonable estimation of $z_{0}^{\text {eff }}$, which can then be used in bulk similarity theory (the logarithmic law with effective parameters under neutral conditions) to estimate SGS turbulent momentum fluxes in numerical models of ABL flow over multiscale terrains. The proportionality parameter $C$ is found to be constant for a given surface elevation field and aerodynamic roughness length $z_{\mathrm{o}}$. For a given surface elevation field, the proportionality parameter increases with increasing roughness of the surface. It is worth pointing out that the proposed simple linear model (Eq. 5) also holds for different realizations of RSOS topography (not shown here), with the same value of the $C$ coefficient.

\section{Summary}

It is known that the spectral density of elevation fields of many natural landscapes shows a -2 slope in a log-log scale over a wide range of spatial scales, from tens of kilometres down to metres (e.g., Rodriguez-Iturbe and Rinaldo 1997; Passalacqua et al. 2006). This noteworthy scaling property led us to investigate its impact on surface momentum flux that results from the interaction between landscapes and the atmospheric boundary layer (ABL). Specifically, we examined how the effective roughness length of multi-scale SGS topography (e.g., unresolved topography in coarser LES or high-resolution weather forecast models) relates to surface information of the topography such as the SGS variance of surface elevation. The multi-scale SGS topography is obtained from a landscape evolution model, the RSOS model (Kim and Kosterlitz 1989). LES with the tuning-free scale-dependent Lagrangian dynamic model (Stoll and Porté-Agel 2006) is used to perform numerical simulations of the ABL flow over a series of multi-scale SGS topographies, and simulation results are used to examine the relation between the effective roughness length of multi-scale SGS topography and the SGS variance of surface elevation.

Large-eddy simulations of ABL flow over surface elevation fields containing different ranges of scales (considered SGS scales in larger scale numerical models) have been performed for three different aerodynamic surface roughness values $z_{0}$. The simulation results 
are used to calculate the resulting effective roughness length $z_{0}^{\text {eff }}$ for each terrain (elevation field and surface roughness combination). By investigating the relation between the quantity $\left(z_{\mathrm{o}}^{\text {eff }}\right)^{2}-z_{\mathrm{o}}^{2}$ and SGS variance of elevation $\sigma_{\mathrm{h}, \mathrm{sgs}}^{2}$, a clear scaling region has been identified for the series of topographies considered. The quantity $\left(z_{0}^{\text {eff }}\right)^{2}-z_{0}^{2}$ is found to increase linearly with the SGS variance of elevation as larger scales are considered, and it starts to converge at some large scale above which the multiscale terrain yields a very small maximum slope (about 6 degrees). The small effect of these scales, characterized by very mild slopes, is consistent with previous simulations results of flow over terrain with multiple hills of the same size. For a given surface elevation field, the magnitude of the slope $C$ of the linear relation between $\left(z_{\mathrm{o}}^{\text {eff }}\right)^{2}-z_{\mathrm{o}}^{2}$ and $\sigma_{\mathrm{h} \text {,sgs }}^{2}$, and therefore the value of the effective roughness length associated with a given terrain, is found to increase with increasing surface roughness of the terrain.

The linear relation between the square of the effective roughness length of multi-scale SGS topography and the SGS variance of the surface elevation field reported in this study could be used in combination with bulk similarity theory (the logarithmic law with effective parameters under neutral stability conditions) to parametrize surface momentum fluxes from natural landscapes. Future research will focus on quantifying the value of the coefficient $C$ as a function of terrain characteristics such as relative steepness of the terrain (e.g., maximum slope) and surface aerodynamic roughness of the surface. The value of $C$ could also be computed dynamically with a procedure similar to the one recently proposed by Anderson and Meneveau (2011) in the context of LES where the effects of the resolved topography are not explicitly represented by the numerical method (e.g., terrain-following coordinates), but parametrized using a drag model.

Acknowledgments This research was supported by the Swiss National Science Foundation (Grant 200021_132122), the US National Science Foundation (Grant ATM-0854766), NASA (Grant NNG06GE256). Computer resources were provided by the Minnesota Supercomputing Institute. We thank Professor Charles Meneveau for very helpful and stimulating comments on this work.

\section{References}

Albertson JD, Parlange MB (1999) Surface length scales in shear stress: implications for land-atmosphere interactions over complex terrain. Water Resour Res 35:2121-2132

Anderson W, Meneveau C (2011) Dynamic roughness model for large-eddy simulation of turbulent flow over multiscale, fractal-like rough surfaces. J Fluid Mech. doi:10.1017/jfm.2011.137

Athanassiadou M, Castro IP (2001) Neutral flow over a series of rough hills: a laboratory experiment. Boundary-Layer Meteorol 101:1-30

Besio S, Mazzino A, Ratto CF (2000) Local log-law of the wall: numerical evidences and reasons. Phys Lett A $275: 152-158$

Brown AR, Wood N (2003) Properties and parameterization of the stable boundary layer over moderate topography. J Atmos Sci 60:2797-2808

Brown AR, Hobson JM, Wood N (2001) Large-eddy simulation of neutral turbulent flow over rough sinusoidal ridges. Boundary-Layer Meteorol 98:411-441

Clark T (1977) A small-scale dynamic model using a terrain-following coordinate transformation. J Comput Phys 24:186-215

De Vries AC, Kustas WP, Ritchie JC, Klaassen W, Menenti M, Rango A, Prueger JH (2003) Effective aerodynamic roughness estimated from airborne laser altimeter measurements of surface features. Int J Remote Sens 24:1545-1558

Ding L, Calhoun RJ, Street RL (2003) Numerical simulation of strongly stratified flow over a three-dimensional hill. Boundary-Layer Meteorol 107:81-114

Flack KA, Schultz MP (2010) Review of hydraulic roughness scales in the fully rough regime. J Fluids Eng. doi:10.1115/1.4001492 
Germano M, Piomelli U, Moin P, Cabot W (1991) A dynamic subgrid-scale eddy viscosity model. Phys Fluids A 3(7):1760-1765

Gong W, Taylor PA, Dörnbrack A (1996) Turbulent boundary-layer flow over fixed aerodynamically rough two-dimensional sinusoidal waves. J Fluid Mech 312:1-37

Grant ALM, Mason PJ (1990) Observations of boundary-layer structure over complex terrain. Q J Roy Meteorol Soc 116:159-186

Henn DS, Sykes RI (1999) Large-eddy simulation of flow over wavy surfaces. J Fluid Mech 383:75-112

Huang J, Turcotte DL (1989) Fractal mapping of digitized images: application to the topography of Arizona and comparisons with synthetic images. J Geophys Res 94(B6):7491-7495

Iizuka S, Kondo H (2004) Performance of various sub-grid scale models in large-eddy simulation of turbulent flow over complex terrain. Atmos Environ 38:7083-7091

Kardar M, Parisi G, Zhang YC (1986) Dynamic scaling of growing interfaces. Phys Rev Lett 56:889-892

Kim JM, Kosterlitz JM (1989) Growth in a restricted solid-on-solid model. Phys Rev Lett 62:2289-2292

Kim HJ, Kim IM, Kim JM (2000) River networks on the slope-correlated landscape. Phys Rev E 62:3121-3124

Krettenauer K, Schumann U (1992) Numerical simulation of turbulent convection over wavy terrain. J Fluid Mech 237:261-299

Kustas WP, Brutsaert W (1986) Wind profile constants in a neutral atmospheric boundary layer over complex terrain. Boundary-Layer Meteorol 34:35-54

Mason PJ, Sykes RI (1979) Flow over an isolated hill of moderate slope. Q J Roy Meteorol Soc 105:383-395

Mason PJ, Thomson DJ (1992) Stochastic backscatter in large-eddy simulations of boundary layers. J Fluid Mech 242:51-78

Meneveau C, Lund T, Cabot W (1996) A Lagrangian dynamic subgrid-scale model of turbulence. J Fluid Mech 319:353-385

Milton SF, Wilson CA (1996) The impact of parameterized subgrid-scale orographic forcing on systematic errors in a global NWP model. Mon Weather Rev 124:2023-2045

Newley TJ (1985) Turbulent airflow over hills. PhD dissertation, University of Cambridge

Park K, Kahng BN (1995) Exact derivation of the Kardar-Parisi-Zhang equation for the restricted solid-onsolid model. Phys Rev E 51:796-798

Passalacqua P, Porté-Agel F, Foufoula-Georgiou E, Paola C (2006) Application of dynamic subgrid-scale concepts from large-eddy simulation to modeling landscape evolution. Water Resour Res 42:W06D11. doi:10.1029/2006WR004879

Pelletier JD (1997) Why is topography fractal? arXiv:physics/9705033v1

Pelletier JD (2007) Fractal behavior in space and time in a simplified model of fluvial landform evolution. Geomorphology 91:291-301

Porté-Agel F, Meneveau C, Parlange MB (2000) A scale-dependent dynamic model for large-eddy simulation: application to a neutral atmospheric boundary layer. J Fluid Mech 415:261-284

Rodriguez-Iturbe I, Rinaldo A (1997) Fractal river basins. Cambridge University Press, New York, 539 pp

Smagorinsky J (1963) General circulation experiments with the primitive equations: I. The basic experiment. Mon Weather Rev 91:99-164

Stoll R, Porté-Agel F (2006) Dynamic subgrid-scale models for momentum and scalar fluxes in large-eddy simulation of neutrally stratified atmospheric boundary layers over heterogeneous terrain. Water Resour Res 42:W01409. doi:10.1029/2005WR003989

Thompson RS (1978) Note on the aerodynamic roughness-length for complex terrain. J Appl Meteorol 17:1402-1403

Walko RL, Cotton WR, Pielke RA (1992) Large-eddy simulations of the effects of hilly terrain on the convective boundary layer. Boundary-Layer Meteorol 58:133-150

Wan F, Porté-Agel F (2011) Large-eddy simulation of stably-stratified flow over a steep hill. Boundary-Layer Meteorol 138:367-384

Wan F, Porté-Agel F, Stoll R (2007) Evaluation of dynamic subgrid-scale models in large-eddy simulations of neutral turbulent flow over a two-dimensional sinusoidal hill. Atmos Environ 41:2719-2728

Weissel JK, Pratson LF, Malinverno A (1994) The length-scaling properties of topography. J Geophys Res 99:13997-14012

Weng W, Chang L, Taylor PA, Xu D (1997) Modelling stably stratified boundary layer flow over low hills. Q J Roy Meteorol Soc 123:1841-1866

Wood N, Mason PJ (1993) The pressure force induced by neutral, turbulent flow over hills. Q J Roy Meteorol Soc 119:1233-1267

Wood N, Brown AR, Hewer FE (2001) Parametrizing the effects of orography on the boundary layer: an alternative to effective roughness lengths. Q J Roy Meteorol Soc 127:759-777

Zagarola MV, Smits AJ (1998) Mean-flow scaling of turbulent pipe flow. J Fluid Mech 373:33-79 